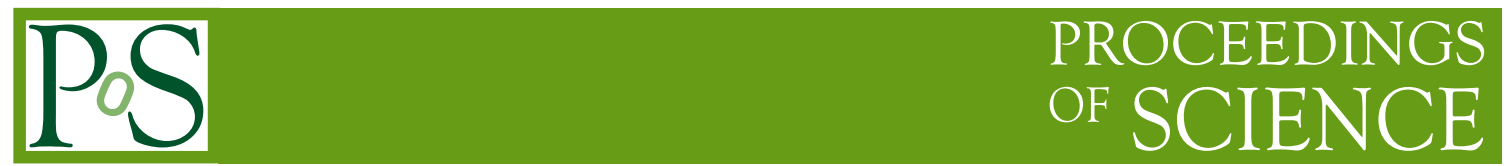

\title{
Hadron Structure
}

\section{Martha Constantinou*}

Department of Physics, University of Cyprus, P.O. Box 20537, 1678 Nicosia, Cyprus

E-mail: marthaceucy.ac.cy

This is a review of recent developments in hadron structure within the framework of Lattice QCD. The main focus is on recent achievements in the evaluation of nucleon quantities, such as the axial charge, electromagnetic form factors, the Dirac and Pauli radii, the quark momentum fraction and the spin content of the nucleon, in view of simulations at pion masses very close to their physical value. A discussion of the systematic uncertainties and the computation of the disconnected contributions using dynamical simulations is also included. Results emerging the propetries of particles other than the nucleon are summarized, highlighting selected hyperon and meson form factors.

32st International Symposium on Lattice Field Theory - LATTICE 2014

June 23 - June 28, 2014

New York, USA

*Speaker. 


\section{Introduction}

Recent progress in the numerical simulation of Lattice QCD has been impressive. This has been due to improvements in the algorithms and the development of new techniques, as well as, the increase in computational power, that have enabled simulations to be carried out at parameters very close to their physical values. The role of Lattice QCD is twofold: to make contact with well determined experimental quantities with their ab initio calculation, as well as, to make predictions on quatities that are not easily accessible in experiment, providing input to phenomenology as well as new input for beyond the Standard Model Physics.

Understanding nucleon structure from first principles is considered a milestone of hadronic physics and numerous experiments have been devoted to its study, starting with the measurements of the electromagnetic form factors initiated more than 50 years ago. Reproducing these key observables within the Lattice QCD formulation is a prerequisite to obtaining reliable predictions on observables that explore Physics beyond the Standard Model. There is a rich experimental program in major facilities (JLab, MAMI, MESA, etc) investigating hadron structure, such are the origin of the nucleon spin, the proton radius puzzle, and searching for new Physics, like $(g-2)_{\mu}$ and dark photon searches.

The $12 \mathrm{GeV}$ upgrade of the Continuous Electron Beam Accelerator Facility at JLab [1] will allow to employ new methods for studying the basic properties of hadrons. Hadron structure has been an essential part of the Physics Program which involves new and interesting high precision experiments, such as nucleon resonance studies with CLAS12, the longitudinal spin structure of the nucleon, meson spectroscopy with low $Q^{2}$ electron scattering, the nucleon generalized parton disctribution functions, high precision measurement of the proton charge radius, and many more.

The experiments on the proton radious have attracted a lot of interest since accurate measurements of the root mean square (r.m.s) charge radius from muonic hydrogen [2] $\left(\left\langle r_{p}^{2}\right\rangle_{\mu H}=0.84 \mathrm{fm}^{2}\right)$ is $7.7 \sigma$ yield a value smaller that the radius determined from elastic e-p scattering and hydrogen spectroscopy $\left(\left\langle r_{p}^{2}\right\rangle_{e p}=0.88 \mathrm{fm}^{2}\right)$ [3]. The $4 \%$ difference in the two measurements is currently not explained. We note that the measurements in the muonic hydrogen experiments are ten times more accurate than other measurements and they are very sensitive to the proton size. In particular, the radius is measured from the energy difference between the $2 \mathrm{P}$ and $2 \mathrm{~S}$ states of the muonic hydrogen [4] and more accurate esxperiments are planned at PSI.

Another interesting topic is the elastic light-by-light scattering $(\gamma \gamma \rightarrow \gamma \gamma)$ which was never observed directly, but only indirectly by its effects on the anomalous magnetic moments of electrons and muons [5]. In addition, photon-photon collisions in 'ultraperipheral' collisions of proton and lead beams have been detected. Recently, there has been a study confirming that light-by-light scattering could be directly detected at LHC, for the first time, at 5.5-14 TeV [6], due to the large 'quasireal' photons fluxes in electromagnetic interactions of protons and lead ions.

The above few examples illustrate that hadron structure is a very rich field of research relevant to new Physics searches. Thus, Lattice QCD does not only provide input to on-going experiments, but also gives guidance to new searches with high-credibility results.

In these proceedings we discuss representative observables probing hadron structure for which there has been recent activity. Topics to be covered include benchmark quantities, such as the nucleon axial charge, electromagnetic form factors, the Dirac and Pauli radii, the quark momentum 
fraction, as well as the nucleon spin, including disconnected contributions. The systematic uncertainties are investigated and where possible we compare with experimental / phenomenological data. Recent results on Generalized Form Factors for other baryons and mesons are also presented, as well as, perspectives and future directions.

\section{Nucleon Sector}

Although the nucleon is the only stable hadron in the Standard Model, its structure is not fully understood. Being one of the building-blocks in the universe, the nucleon provides an extremely valuable laboratory for studying strong dynamics providing important input that can also shed light in new Physics searches. There have been numerous recent lattice QCD results on nucleon observables. Here, we discuss selected achievements, as well as, challenges involved in these computations.

In a nutshell, in the evaluation of nucleon matrix elements in lattice QCD there are two type of diagrams entering shown in Fig. 1. The disconnected diagram has been neglected in most of the studies because it is very noisy and expensive to compute. During the last few years a number of groups are studying various techniques for its computation and first results already appear in the literature [7-10].
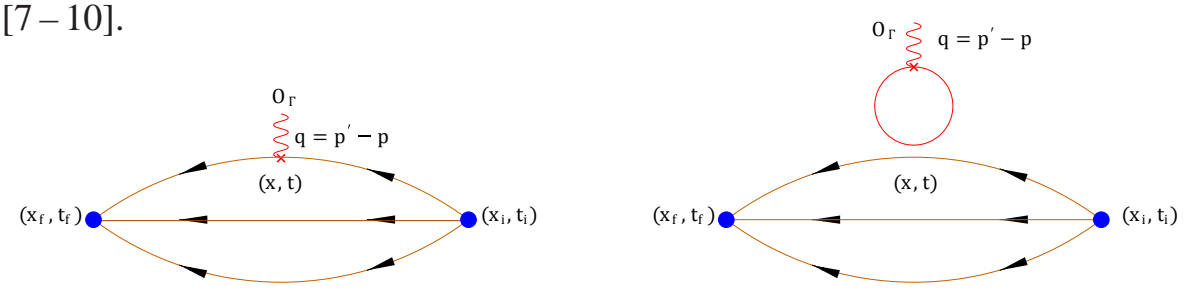

Figure 1: Connected (left) and disconnected (right) contributions to the nucleon three-point function.

In the computation of nucleon matrix elements one needs appropriate two- and three-point correlation functions defined as:

$$
\begin{aligned}
G^{2 p t}\left(\vec{q}, t_{f}\right) & =\sum_{\vec{x}_{f}} e^{-i \vec{x}_{f} \cdot \vec{q}} \Gamma_{\beta \alpha}^{0}\left\langle J_{\alpha}\left(\vec{x}_{f}, t_{f}\right) \bar{J}_{\beta}(0)\right\rangle, \\
G_{\mathscr{O}}^{3 p t}\left(\Gamma^{\mu}, \vec{q}, t_{f}\right) & =\sum_{\vec{x}_{f}, \vec{x}} e^{i \vec{x} \cdot \vec{q}} e^{-i \vec{x}_{f} \cdot \vec{p}^{\prime}} \Gamma_{\beta \alpha}^{\mu}\left\langle J_{\alpha}\left(\vec{x}_{f}, t_{f}\right) \mathscr{O}(\vec{x}, t) \bar{J}_{\beta}(0)\right\rangle .
\end{aligned}
$$

The projectors $\Gamma^{\mu}$ are defined as $\Gamma^{0} \equiv \frac{1}{4}\left(1+\gamma_{0}\right), \Gamma^{k} \equiv \Gamma^{0} \cdot \gamma_{5} \cdot \gamma_{k}$. Other $\Gamma$-variations can be employed, in order to compute the quantities of interest. The lattice data are extracted from dimensionless ratio of the two- and three-point correlation functions:

$$
R_{\mathscr{O}}\left(\Gamma, \vec{q}, t, t_{f}\right)=\frac{G_{\mathscr{O}}^{3 p t}(\Gamma, \vec{q}, t)}{G^{2 p t}\left(\overrightarrow{0}, t_{f}\right)} \times \sqrt{\frac{G^{2 p t}\left(-\vec{q}, t_{f}-t\right) G^{2 p t}(\overrightarrow{0}, t) G^{2 p t}\left(\overrightarrow{0}, t_{f}\right)}{G^{2 p t}\left(\overrightarrow{0}, t_{f}-t\right) G^{2 p t}(-\vec{q}, t) G^{2 p t}\left(-\vec{q}, t_{f}\right)}} \underset{\substack{t \\ t-t \rightarrow \infty \\ t-t_{i} \rightarrow \infty}}{\rightarrow} \Pi(\Gamma, \vec{q}) .
$$

The above ratio is considered optimized since it does not contain potentially noisy two-point functions at large separations and because correlations between its different factors reduce the statistical noise. The most common method to extract the desired matrix element is to look for a plateau with respect to the current insertion time, $t$ (or, alternatively, the sink time, $t_{f}$ ), which should be located at a time well separated from the creation and annihilation times in order to ensure single state dominance. To establish proper connection to experiments we apply renormalization which, for the quantities discussed in this review, is multiplicative: 


$$
\Pi^{R}(\Gamma, \vec{q})=Z_{\mathscr{O}} \Pi(\Gamma, \vec{q}) .
$$

Finally, the nucleon matrix elements can be parameterized in terms of Generalized Form Factors (GFFs). As an example we take the axial current insertion which decomposes into two Lorentz invariant Form Factors (FFs), the axial $\left(G_{A}\right)$ and pseudoscalar $\left(G_{p}\right)$ :

$\left\langle N\left(p^{\prime}, s^{\prime}\right)\left|\bar{\psi}(x) \gamma_{\mu} \gamma_{5} \psi(x)\right| N(p, s)\right\rangle=i\left(\frac{m_{N}^{2}}{E_{N}\left(\mathbf{p}^{\prime}\right) E_{N}(\mathbf{p})}\right)^{1 / 2} \bar{u}_{N}\left(p^{\prime}, s^{\prime}\right)\left[G_{A}\left(q^{2}\right) \gamma_{\mu} \gamma_{5}+\frac{q_{\mu} \gamma_{5}}{2 m_{N}} G_{p}\left(q^{2}\right)\right] u_{N}(p, s)$,

where $q^{2}$ is the momentum transfer in Minkowski space (hereafter, $Q^{2}=-q^{2}$ ).

In these proceedings I will mostly consider the flavor isovector combination for which the disconnected contribution cancels out; strictly speaking, this happens for actions with exact isospin symmetry. Another advantage of the isovector combination is that the renormalization simplifies considerably.

\subsection{Nucleon Axial Charge}

One of the fundamental nucleon observables is the axial charge, $g_{A} \equiv G_{A}(0)$, which is determined from the forward matrix element of the axial current. $g_{A}^{q}$ gives the intrinsic quark spin in the nucleon. It governs the rate of $\beta$-decay and has been measured precisely. In the lattice QCD it can be determined directly from the evaluation of the matrix element and thus, there is no ambiguity asocciated to fits. For this reasons, $g_{A}$ is an optimal benchmark quantity for hadron structure computations. It is thus essential for lattice QCD to reproduce its experimental value or if a deviation is observed to understand its origin.

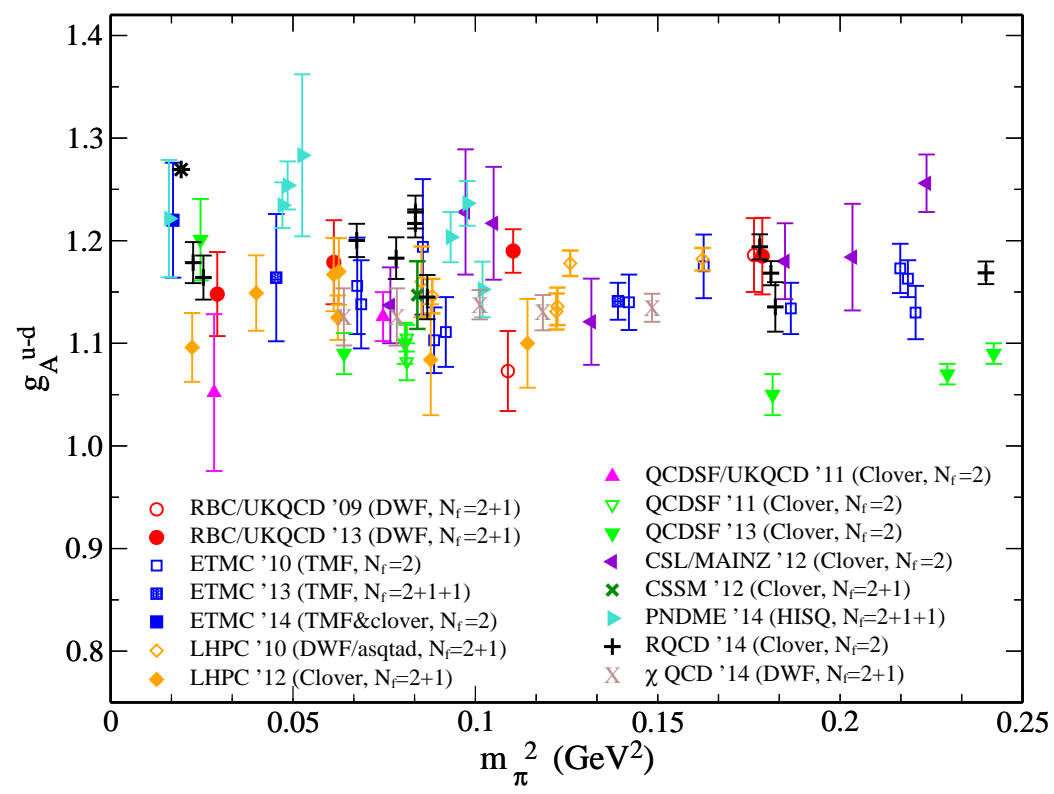

Figure 2: Collection of lattice results for $g_{A}$. In chronological order these correspond to: $N_{f}=2+1 \mathrm{DWF}$ (RBC/UKQCD [11, 12], RBC/UKQCD [13], $\chi \mathrm{QCD}$ [14]), $N_{f}=2+1$ DWF on asqtad sea (LHPC [15]), $N_{f}=2$ TMF (ETMC [16]), $N_{f}=2$ Clover (QCDSF/UKQCD [17], CLS/MAINZ [18], QCDSF [19], RQCD [20, 21]), $N_{f}=1+2$ Clover (LHPC [22], CSSM [23]), $N_{f}=2+1+1$ TMF (ETMC [24]), $N_{f}=2+1+1$ HISQ (PNDME [25, 26]), $N_{f}=2 \mathrm{TMF}$ with Clover (ETMC [27]). The asterisk is the experimental value. 
There are numerous computations from many collaborations and some selected results are shown in Fig. 2 as a function of the squared pion mass. These results have been obtained using dynamical gauge field configurations with $\mathscr{O}(a)$-improved lattice QCD actions, namely Domain Wall Fermions (DWF), Staggered, Clover, Twisted Mass Fermions (TMF) and HISQ fermions [11, $12,15-17,8,18,22,23,19,24,25,13,20]$. For a fair comparison we include only results obtained from the plateau method without any volume corrections. The latest achievement of the Lattice Community are the results at the physical point for which there is no necessity of chiral extrapolation eliminating an up to now uncontrolled extrapolation. The ones at the two lowest values of the pion mass correspond to PNDME (128 MeV) [26] and ETMC (130 MeV) [27], and are in agreement with the experimental value: $g_{A}^{\exp }=0.2701(25)$ [28]. Of course the statistical errors are still large and it is necessary to increase the statistics and study the volume and lattice spacing dependence before finalizing these results. One should also keep in mind that the results shown in Fig. 2 are at a given lattice spacing and volume, and each Collaboration addresses systematic effects in different ways. We will comment later on this issue.

- Cut-off effects: For a proper continuum extrapolation one requires three lattice spacings, which is computationally very costly, especially as we approach the physical point. Thus, it is crucial to investigate cut-off effects using an ensemble with a heavier pion mass. Such a study was done with TMF [16], Clover [21] and HISQ [26] fermions and the results are shown in Fig. 3. Data with the same symbol correspond to similar pion mass, spanning from $\sim 220$ to $480 \mathrm{MeV}$. These results show that with lattice spacings up to about 0.1 fm lattice artifacts are small compared to the statistical accuracy.

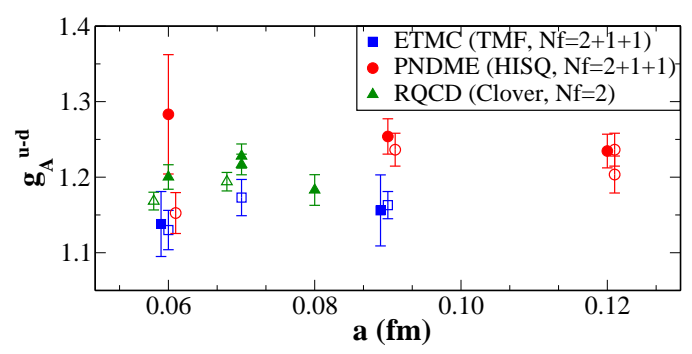

Figure 3: The axial charge as a function of the lattice spacing. The data correspond to: a. TM fermions (ETMC): open/filled symbols for $m_{\pi} \sim 465 / 260 \mathrm{MeV}$, b. HISQ fermions (PNDME): open/filled symbols for $m_{\pi} \sim 310 / 220 \mathrm{MeV}$, c. Clover fermions (RQCD): open/filled symbols for $m_{\pi} \sim 420 / 280 \mathrm{MeV}$. Points have been horizontally shifted for clarity.

- Excited states contamination: The interpolating field used to create a hadron of given quantum numbers couple in addition to the excited states. While for two-point functions identification of the ground is straight forward for three-point functions it is more saddle. The most common approach is the so called plateau method in which one probes the large Euclidean time evolution of the ratio in Eq. (2.3)

$$
R_{\mathscr{O}}\left(\Gamma, t_{i}, t, t_{f}\right) \underset{\substack{\left(t_{f}-t\right) \Delta>>1 \\\left(t-t_{i}\right) \Delta>>1}}{\rightarrow} \mathscr{M}\left[1+\alpha e^{-\left(t_{f}-t\right) \Delta\left(p^{\prime}\right)}+\beta e^{-\left(t-t_{i}\right) \Delta(p)}+\cdots\right] .
$$


As can be seen in the above equation, the excited states contributions fall exponentially with the sink-insertion $\left(t_{f}-t\right)$ and insertion-source $\left(t-t_{i}\right)$ time separation. So, it is possible to reduce the unwanted excited states contamination by increasing the source-sink separation, but this comes with a cost of increased statistical noise.

Alternatively, the matrix element may be obtained by performing a 2-state fit to account for contributions from the first excited state. One can also perform simultaneous fits on data for different sink-source separations

$$
\begin{aligned}
\mathscr{C}_{\Gamma}^{(3), T}\left(t_{i}, t, t_{f} ; \vec{p}_{i}, \vec{p}_{f}\right) \approx\left|\mathscr{A}_{0}\right|^{2}\left\langle 0\left|O_{\Gamma}\right| 0\right\rangle e^{-M_{0}\left(t_{f}-t_{i}\right)}+\left|\mathscr{A}_{1}\right|^{2}\left\langle 1\left|O_{\Gamma}\right| 1\right\rangle e^{-M_{1}\left(t_{f}-t_{i}\right)}+ \\
\mathscr{A}_{0} \mathscr{A}_{1}^{*}\left\langle 0\left|O_{\Gamma}\right| 1\right\rangle e^{-M_{0}\left(t-t_{i}\right)} e^{-M_{1}\left(t_{f}-t\right)}+\mathscr{A}_{0}^{*} \mathscr{A}_{1}\left\langle 1\left|O_{\Gamma}\right| 0\right\rangle e^{-M_{1}\left(t-t_{i}\right)} e^{-M_{0}\left(t_{f}-t\right)}
\end{aligned}
$$

where $M_{0}$ and $M_{1}$ are the masses of the ground and first excited state, respectively, and $\mathscr{A}_{0} \mathscr{A}_{1}$ are the corresponding amplitudes.

A third method is the so-called summation method in which we sum the ratio from the source to the sink ${ }^{1}$. This way, the excited state contaminations are suppressed by exponentials decaying with $\left(t_{f}-t_{i}\right)$ rather than $\left(t_{f}-t\right)$ and $\left(t-t_{i}\right)$. However, one needs the slope of the summmed ratio:

$$
\sum_{t=t_{i}}^{t_{f}} R\left(t_{i}, t, t_{f}\right)=\text { const. }+\mathscr{M}\left(t_{f}-t_{i}\right)+\mathscr{O}\left(e^{-\left(\left(t_{f}-t_{i}\right) \Delta\left(p^{\prime}\right)\right)}\right)+\mathscr{O}\left(e^{-\left(\left(t_{f}-t_{i}\right) \Delta(p)\right)}\right) .
$$

All the aforementioned methods have been applied in the extraction of $g_{A}$ and some of the works are presented here. In a study by RQCD [21] at $m_{\pi} 300 \mathrm{MeV}$, no change of $g_{A}$ has been seen by varying the separation from $0.5-1.2 \mathrm{fm}$ (see Fig. $4 \mathrm{a}$ ); this is also confirmed by a high precision study at $m_{\pi} \sim 370 \mathrm{MeV}$ using TMF [29] in which the source-sink separation takes values within the range 0.9-1.6 fm [29].

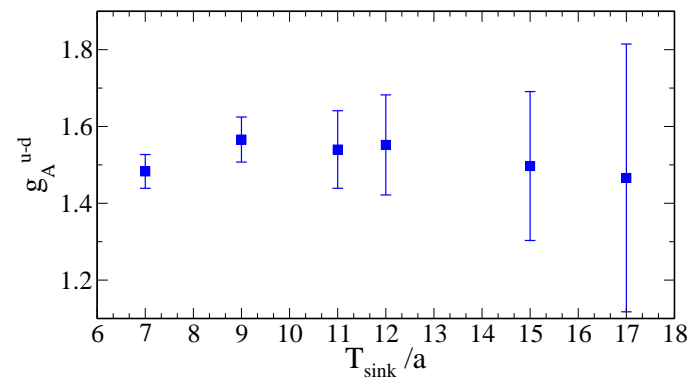

(a)

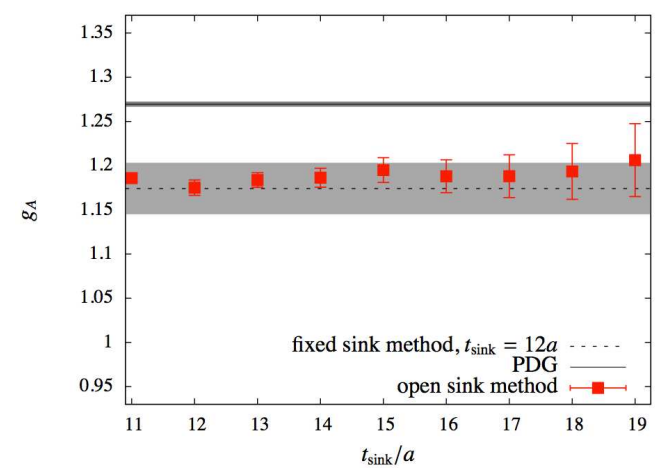

(b)

Figure 4: The axial charge as a function of the source-sink separation for (a). $N_{f}=2$ Clover fermions at $m_{\pi} 300 \mathrm{MeV}$ (RQCD [21]), and (b). $N_{f}=2+1+1$ TMF (ETMC [29]).

Studying the summed ratio using TMF no curvature in the slope is observed and the result extracted for $g_{A}$ agrees with the plateau method as can be seen in Fig 5a. The 2-state analysis of PNDME [25] shown in Fig. 5b (purple points) agrees with the corresponding single state fit (orange points), as well as with the 2-state simultaneous fit using the lattice data on different separations (blue band).

\footnotetext{
${ }^{1}$ The result also holds by excluding the source and sink points to avoid contact terms.
} 


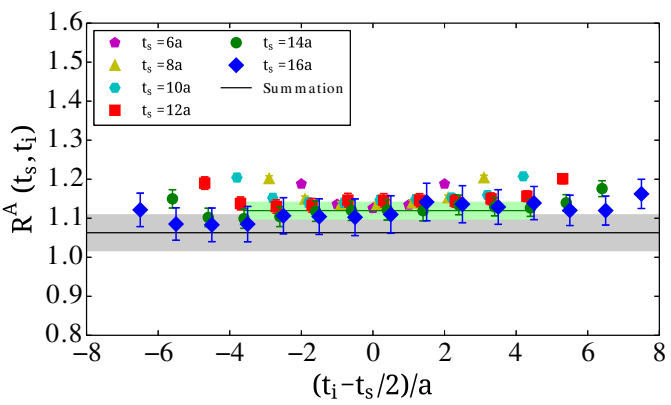

(a)

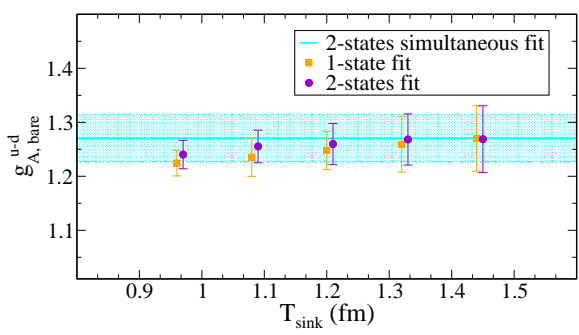

(b)

Figure 5: (a). The ratio of the axial charge for $N_{f}=2+1+1$ TMF (ETMC [24]), (b). Bare values for $g_{A}$ as a function of the source-sink separation using $N_{f}=2+1+1$ HISQ fermions at $m_{\pi}=310 \mathrm{MeV}$.

Recently the Feynman-Hellmann approach was utilized for the computation of $g_{A}$ by CSSM/ QCDSF/ UKQCD [30] at $m_{\pi}=470 \mathrm{MeV}$, which is based on the introduction of an external spin operator to the fermion part of the action:

$$
S \rightarrow S(\lambda)=S+\lambda \sum_{x} \bar{q}(x) i \gamma_{5} \gamma_{3} q(x) .
$$

The axial charge can then be extracted by the linear response of the hadron energies:

$$
\Delta q=\left.\frac{\partial E(\lambda)}{\partial \lambda}\right|_{\lambda=0}=\frac{1}{2 M}\left\langle N\left|\bar{q} i \gamma_{5} \gamma_{3} q\right| N\right\rangle .
$$

The authors find improved statistical accuracy compared to the standard method. It would be interesting to test this approach in observables highly contaminated by excited states, since it is believed that this method is less susceptible to excited states contributions.

We note that all high statistics studies of systematic uncertainties have been performed at relatively large values of the pion mass. It is thus essential to also perform similar investigations at values of the pion mass closer to the physical one. Given that the signal to noise error decreases exponentially as the pion mass decreases: one needs to increase considerably the number of independent measurements leading to increase computational cost. Thus, noice reduction methods are highly valuable. RBC/UKQCD has implemented a new class of covariant approximation averaging techniques, called All-Mode-Averaging (AMA) [31] which allows for a significant decrease of the statistical error over conventional methods at reduced computational cost. The AMA technique achieves an increase of the number of measurement, $N_{\text {meas }}$, at a low computational cost, by constructing an improved operator which is built from a low-precision and thus cheap part, and a correction term, to compensate the bias introduced by the approximate measurements:

$$
\left\langle\mathscr{O}^{\text {impr }}\right\rangle=\left\langle\mathscr{O}^{\text {approx }}\right\rangle+\left\langle\mathscr{O}^{\text {rest }}\right\rangle, \quad \mathscr{O}^{\text {rest }}=\mathscr{O}^{\text {exact }}-\mathscr{O}^{\text {approx }} .
$$

The approximate operator has the same covariance properties as the original one, but a much smaller construction cost and thus $\mathscr{O}^{\text {impr }}$ has smaller statistical errors without additional computational cost. In other words, the AMA allows to increase statistics using a large number of sloppy measurements and a small number of exact ones:

$$
O_{\text {AMA }}=\frac{1}{N_{\text {apprx }}} \sum_{i=1}^{N_{\text {apprx }}} O_{\text {apprx }}^{i}+\frac{1}{N_{\text {exact }}} \sum_{j=1}^{N_{\text {exact }}}\left(O_{\text {exact }}^{j}-O_{\text {apprx }}^{j}\right) .
$$


It was shown that the error of the combined result depends highly on the number of approximate measurements. For the case of $g_{A}$ a speed up of $\sim 5-20$ times is reported using $N_{f}=2+1 \mathrm{DWF}$ configurations on a $24^{3} \times 48$ lattice, and up to 10-30 times for a larger lattice, $32^{3} \times 64$ [31].

- Finite volume effects: To assess volume effects we plot $g_{A}$ against $L m_{\pi}$, in Fig. 6 . In order for the data to be distinguishable we restrict to pion mass of about $300 \mathrm{MeV}$. We highlight results at almost physical values of the pion mass by the black filled symbols. These come from a range of collaborations, namely: PNDME [26] at $m_{\pi}=128 \mathrm{MeV}$, ETMC [27] at $m_{\pi}=130 \mathrm{MeV}$, LHPC [22] at $m_{\pi}=149 \mathrm{MeV}$, RQCD [21] at $m_{\pi}=150 / 157 \mathrm{MeV}$, QCDSF [19, 17] at $m_{\pi}=170 \mathrm{MeV}$ and $\mathrm{RBC}[32]$ at $m_{\pi}=170 \mathrm{MeV}$.

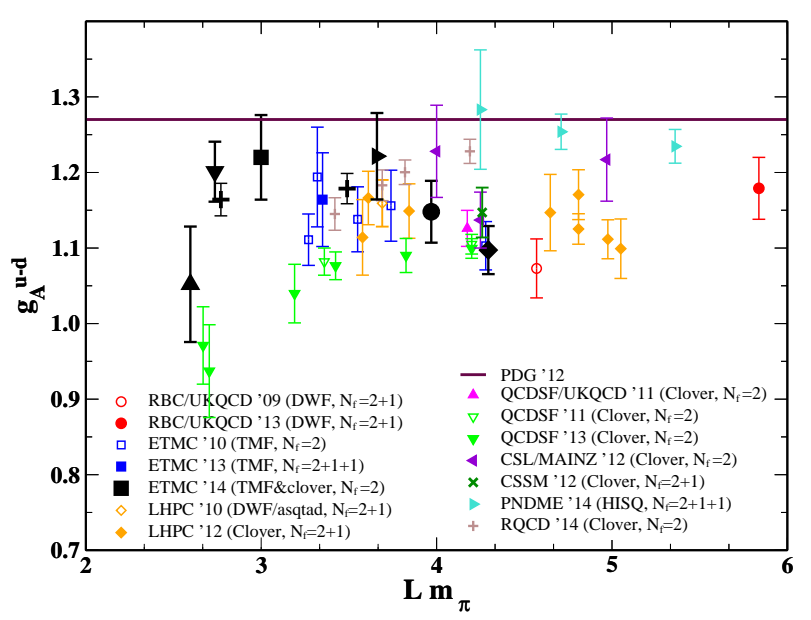

Figure 6: Summary of lattice results for $g_{A}$ as a function of the finite scaling parameter $L m_{\pi}$. Undefined black symbols correspond to the same action as colored symbols. See caption of Fig. 2 for references.

One immediately observes that data at $L m_{\pi} \sim 3,3.7$ agree with experiment, while data at $L m_{\pi}>4$ are below the experimental point. This behaviour is puzzling and further studies are required to clarify the volume dependence.

In summary, based on current results on the axial charge, we conclude that cut-off effects are small, at least for $a \leq 0.1 \mathrm{fm}$, and no indication of significant excited state contamination has been observed indicating that sink-source time separation of about $1 \mathrm{fm}$ is sufficient. No clear conclusion can be extracted regarding finite volume effects that need further investigation. It is worth stressing however that the preliminary value of $g_{A}$ determined by ETMC close to the physical point with $L m_{\pi} \sim 3$ and $a<0.1 \mathrm{fm}$ is in agreement with the experimental value.

\subsection{Nucleon Electromagnetic Form Factors}

The matrix element of the vector current decomposes into the Dirac and Pauli FFs:

$$
\left\langle N\left(p^{\prime}, s^{\prime}\right)\left|\gamma_{\mu}\right| N(p, s)\right\rangle \sim \bar{u}_{N}\left(p^{\prime}, s^{\prime}\right)\left[F_{1}\left(q^{2}\right) \gamma_{\mu}+F_{2}\left(q^{2}\right) \frac{i \sigma^{\mu \rho} q_{\rho}}{2 m_{N}}\right] u_{N}(p, s) .
$$

The most recent study by LHPC at $m_{\pi}=149 \mathrm{MeV}$, with statistics exceeding 7000 measurements, explores three values of the source - sink separation, on which the summation method is also 
applied. As an example, we show the Dirac form factor $F_{1}\left(Q^{2}\right)$ in Fig. 7a, while in Fig. 7b we compare results for $F_{1}$ and $F_{2}$ using Clover fermions $\left(m_{\pi}=149 \mathrm{MeV}\right)$ [33] and TMF $\left(m_{\pi}=130\right.$ $\mathrm{MeV})[34]$.

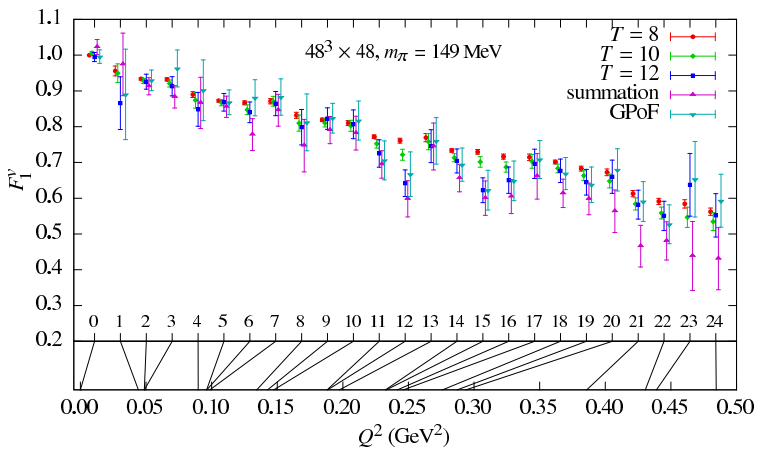

(a)

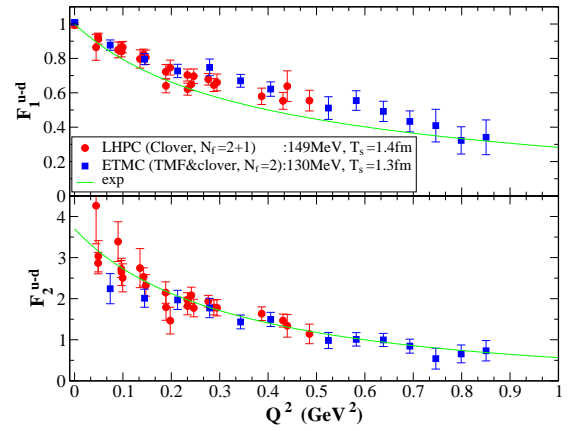

(b)

Figure 7: (a). Isovector $F_{1}$ extracted from different methods for $N_{f}=2$ Clover fermions at $m_{\pi}=149$ $\mathrm{MeV}$ [33] (b). Comparison of the Dirac and Pauli form factors for Clover and TM fermions at pion mass close to the physical point, plotted against $Q^{2}$.The solid line is J. Kelly's parametrization of the experimental data [35].

We find a nice agreement between the two discretizations and the slope has been improved compared to data at higher pion masses. Of course the statistical errors are still large, and before reaching to conclusions the statistics must be increased; the AMA technique could be extremelly usefull in the error reduction, as can be seen in Fig. 8. The data correspond to $N_{f}=2+1 \mathrm{DWF}$ at $m_{\pi}=170 \mathrm{MeV}$ [36]. The utilization of the AMA technique on a $32^{3} \times 64$ lattice led to roughly a factor of 20 improvement in the computational efficiency, and to a dramatic reduction in the statistical errors.

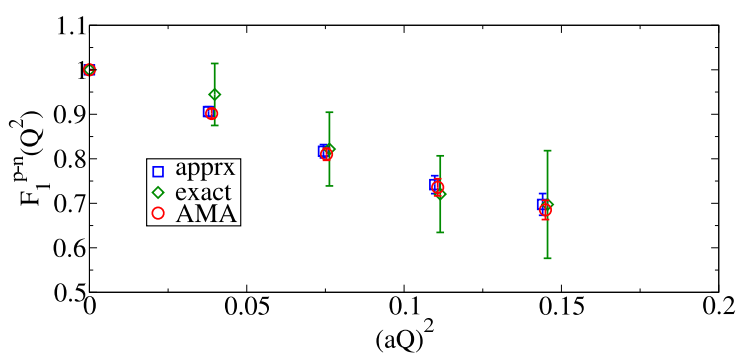

Figure 8: The isovector Dirac form factor for $N_{f}=2+1 \mathrm{DWF}$ at $m_{\pi}=170 \mathrm{MeV}$ [36]. The points have been slightly shifted for clarity.

Preliminary results for disconnected contributions are available by LHPC [37] for the Sachs FFs:

$$
G_{E}\left(Q^{2}\right)=F_{1}\left(Q^{2}\right)-\frac{Q^{2}}{4 m_{N}^{2}} F_{2}\left(Q^{2}\right), \quad G_{M}\left(Q^{2}\right)=F_{1}\left(Q^{2}\right)+F_{2}\left(Q^{2}\right),
$$

using hierarchical probing [38] for the computation of the quark loops. The gain of this method depends on the observable and for the electromagnetic FFs is significant, leading to results with very impresive accuracy at various values of the momentum transfer. The hierarchical probing is a spatial dilution method using a sequence of deterministic orthonormal vectors, called Hadamard, 
built out of 1's and -1's in a specific order. The 'level of dilution' is increased gradually, at any stage of the computation, while reusing existing data. The method improves the stochastic estimator: $\operatorname{Tr}\left[A^{-1}\right]=E\left\{z^{\dagger} A^{-1} z\right\}$ ( $z$ : noise vector), using the fact that $A_{i, j}^{-1}$ is dense but decays exponentially as the distance, $k$, between the sites $i, j$, i.e. $|i-j|$ increases. The optimal distance $k$ for $A_{i, j}^{-1} \approx 0$ is obtained using probing, where the results from level $i-1$ is used at level $i$. The multi coloring of sites is done hierarchically and the bias is removed by using a random starting vector. A factor of 10 speed up in the computational cost over standard approach has been reported on a $32^{3} \times 64$ Clover lattice at the strange quark mass.

The connected and disconnected contributions to the proton Sachs FFs, as well as their ratio are shown in Fig. 9 for $N_{f}=2+1$ Clover fermions at a pion mass of $317 \mathrm{MeV}$, and the statistics used is $\sim \mathscr{O}(100000)$. It is interesting to see that the disconnected contribution of $G_{E}$ increases the total value, while the total $G_{M}$ decreases. In both cases, the disconnected contribution are approximately $0.5 \%$ of the connected contribution.
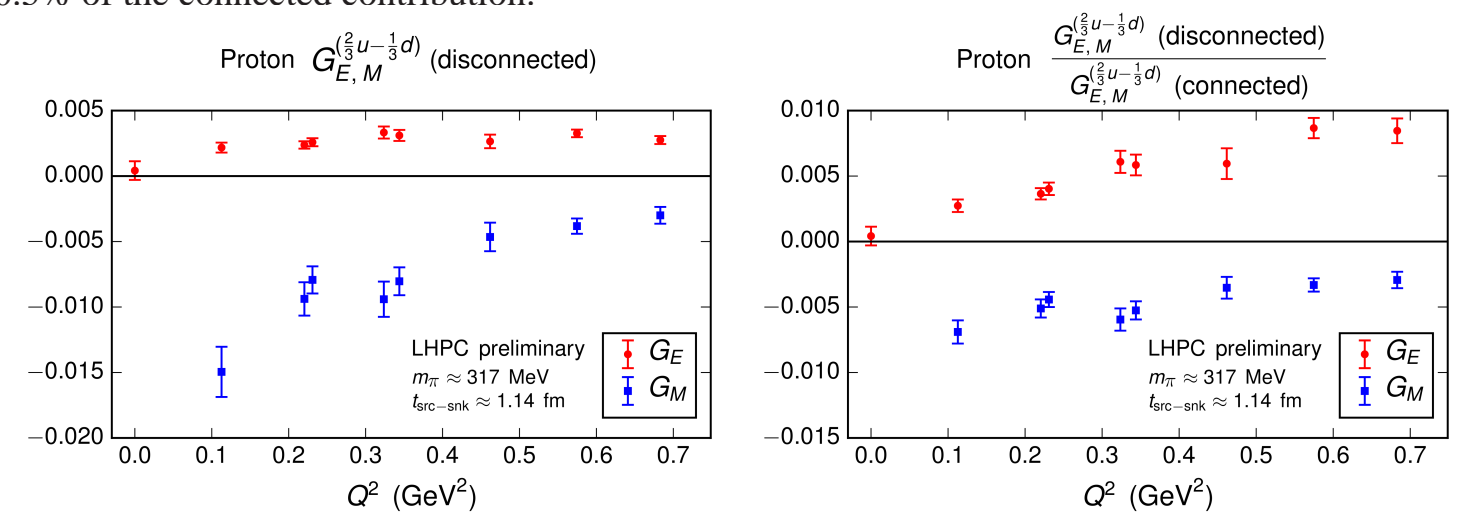

Figure 9: Results for the proton Sach FFs using $N_{f}=2+1$ Clover fermions at $m_{\pi}=317 \mathrm{MeV}$ [37].

\subsection{Dirac \& Pauli radii}

In the non-relativistic limit the slope of the electromagnetic form factors, $F_{1}, F_{2}$, at zero momentum transfer is characterized by the Dirac, $r_{1}$, and Pauli, $r_{2}$, radii of charge:

$$
F_{i}\left(Q^{2}\right) \sim F_{i}(0)\left(1-\frac{1}{6} Q^{2}\left\langle r_{i}^{2}\right\rangle+\mathscr{O}\left(Q^{4}\right)\right), \quad\left\langle r_{i}^{2}\right\rangle=-\left.\frac{6}{F_{i}\left(Q^{2}\right)} \frac{d F_{i}\left(Q^{2}\right)}{d Q^{2}}\right|_{Q^{2}=0} .
$$

To determine the slope we fit the form factors using a dipole function, and therefore, the r.m.s radii can be obtained from the values of the dipole mass:

$$
F_{i}\left(Q^{2}\right) \sim F_{i}(0) /\left(1+Q^{2} / m_{i}^{2}\right)^{2}, \quad\left\langle r_{i}^{2}\right\rangle=12 / m_{i}^{2} .
$$

Input on these radii from Lattice $\mathrm{QCD}$ is significant due to the persisting descrepancy between experiments involving electrons and muons [2,3]. A collection of lattice results for $\left\langle r_{1}^{2}\right\rangle$ appears in the left panel of Fig. 10 and for $\left\langle r_{2}^{2}\right\rangle$ in the right panel. Overall we find a good agreement among different lattice discretizations and as we approach the physical point we observe an increasing trend of the data which could be justified be a logarithmic chiral behavior. The Pauli radius appears to have larger statistical errors compared to the Dirac radius which is expected since, in the dipole fit there is an additional parameter, $F_{2}(0)$, that cannot be obtained directly from the lattice data. Since the estimation of the radii is strongly dependent on the small momenta, we need access to momenta closer to zero, and thus, simulations of nucleons of nucleons in larger boxes are necessary. 

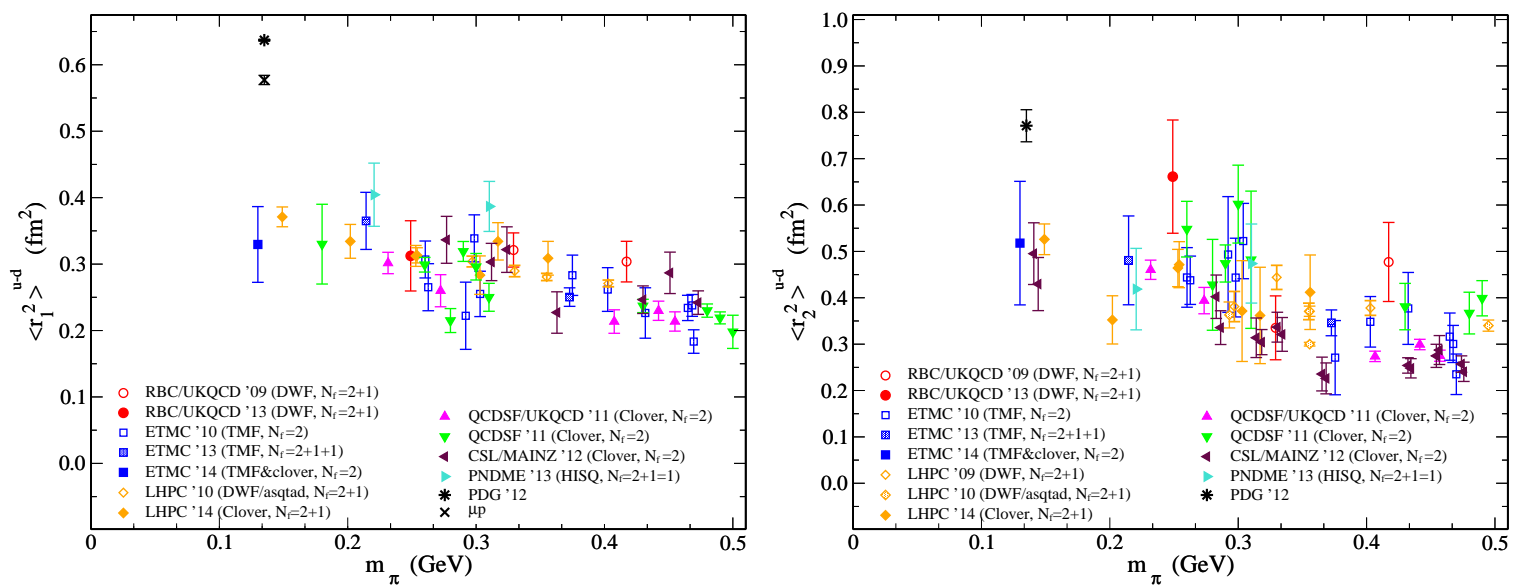

Figure 10: Dirac (left) and Pauli (right) radii as a function of $m_{\pi}$. The lattice data correspond to: $N_{f}=2+1 \mathrm{DWF}(\mathrm{RBC} / \mathrm{UKQCD}[12,39]), N_{f}=2 \mathrm{TMF}$ (ETMC [40]), $N_{f}=2+1 \mathrm{DWF}\left(\mathrm{LHPC}\right.$ [41]), $N_{f}=2+1$ DWF on asqtad sea (LHPC [15]), $N_{f}=2$ Clover (QCDSF/UKQCD [17], QCDSF [42], CLS/MAINZ [43]), $N_{f}=2+1+1$ TMF (ETMC [24]), $N_{f}=2+1+1$ HISQ (PNDME [25]), $N_{f}=2+1$ Clover (LHPC [33]), $N_{f}=2$ TMF with Clover (ETMC [34]). The experimental points have been taken from Refs. [2, 3].

In the left panel of Fig. 11 we plot $r_{1}$ for a range of pion mass as the sink-source separation increases. The study is carried out by LHPC [33] and ETMC [34] and it include the result of the summation method. There is a clear upward tendency as the sink-source time separation increases. Although the results from the summation method agree with the value extracted from the plateau method for the largest sink-source time separation, the errors on the results at lowest values of the pion masses are still large and currently do not allow to reach a definite conclusion.
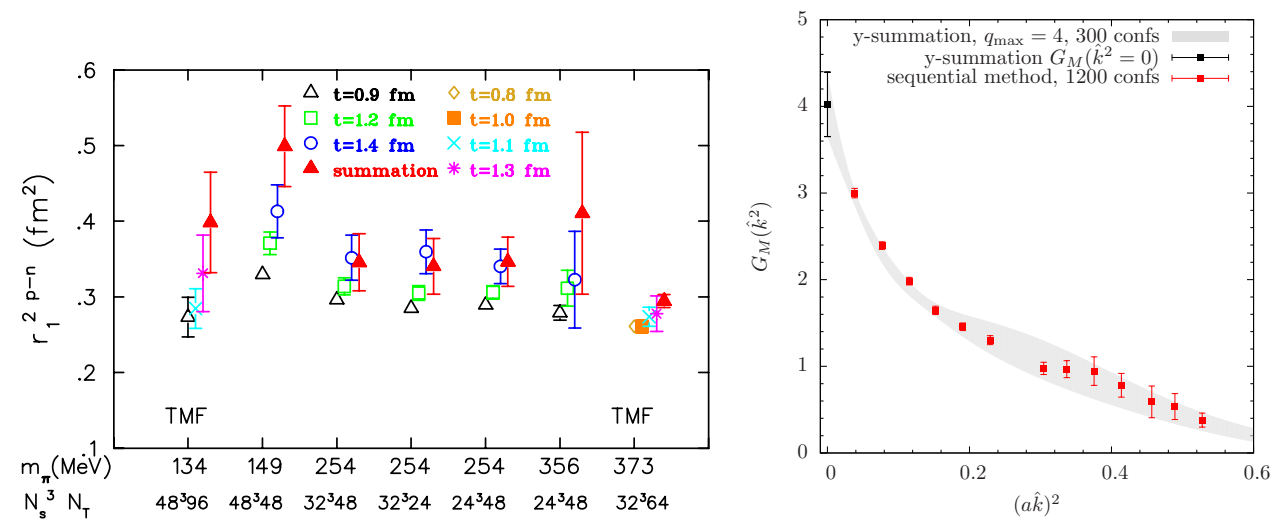

Figure 11: Left: Isovector $\left\langle r_{1}^{2}\right\rangle$ for various ensembles and different source-sink separations [33, 34]. Right: Isovector $G_{M}$ for TMF extracted from a position space method [44].

In order to extract the anomalous magnetic moment one needs to fit the $Q^{2}$-dependence of $G_{M}$. Typically one employs a dipole form to extrapolate at $Q^{2}=0$ introducing a model-dependence. Exploratory studies based on a position space method can yield $G_{M}(0)$ directly without having to perform a fit. This method involves taking the derivative of the relevant correlator with respect to the momentum, allowing access to zero momentum data. Thus, there is no need to assume a functional form for the momentum dependence. Such a study is performed for $G_{M}$ [44] and the results are shown in the right panel of Fig. 11. 


\subsection{Quark momentum fraction}

Another important observable of hadron structure is the quark momentum fraction. This is directly related to the first moment of the unpolarized structure function through the operator product expansion. It can be extracted from the forward matrix element of the one-derivative vector current, and it is a scheme and scale dependent quantity. Experimentally it is measured in Deep Inelastic Scattering where phenomenological input is required in order to extract it from measurements. Fig. 12 shows results on the isovector momentum fraction converted to $\overline{\mathrm{MS}}$ scheme at a scale of $2 \mathrm{GeV}$. It has been known for some time that the lattice QCD value using different discretizations is larger than the experimental one. It is worth mentioning that the phenomenological value extracted from different analyses (see Refs. [56-61] of Ref. [24]) shows a spread, which, however, is significantly smaller than the discrepancy shown by the lattice data. Nevertheless, lattice results close to the physical point obtained from different discretizations are in agreement. These correspond to source-sink separation of $\sim 1-1.2 \mathrm{fm}$ which, as discussed below, might not be large enough.

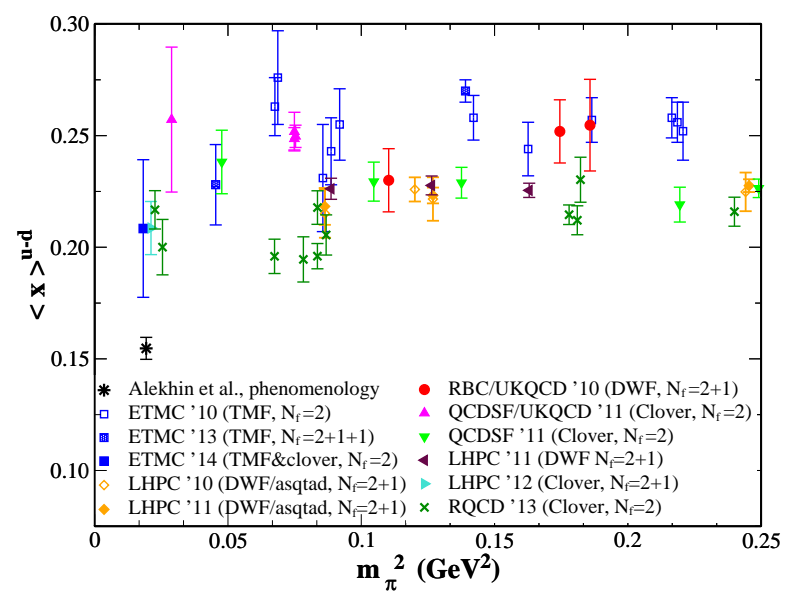

Figure 12: Recent lattice results on the isovector unpolarized moment, $\langle x\rangle_{u-d}$, as a function of the squared pion mass. The lattice data correspond to: $N_{f}=2$ TMF (ETMC [45]), $N_{f}=2+1$ DWF on asqtad sea (LHPC [15, 46]), $N_{f}=2+1$ DWF (RBC/UKQCD [47], LHPC [46]), $N_{f}=2$ Clover (QCDSF/UKQCD [17], QCDSF [48], RQCD [20, 21]), $N_{f}=1+2$ Clover (LHPC [49]), $N_{f}=2+1+1$ TMF (ETMC [24]), $N_{f}=2$ TMF with Clover (ETMC [27]). The phenomenological value shown is 0.1646(27) [50].

A number of studies were undertaken to examine the role of excited states in the extraction of $\langle x\rangle_{u-d}$ and all works corroborate that this observable suffers from excited states contamination, as demonstrated in Fig. 13. Various pion masses have been explored (149-373 MeV), as well as the plateau and summation (Figs. 13b - 13d) methods. The data of Figs. 13a - 13d correspond to lattice spacings $0.082,0.05,0.06,0.116 \mathrm{fm}$, respectively and the source-sink separation for each plot varies within the range: [0.94-1.87], [0.6-1.4], [0.63-1.05] and [0.9-1.4] fermi. It is shown that excited states contamination are accounted for a decrease of about $10 \%$ in the value of $\langle x\rangle_{u-d}$ as compared to the value extracted using sink-source separations of about $1 \mathrm{fm}$. Thus, one should seek for convergence with increasing the source-sink separation and agreement with the summation method. Only then we will have confidence in the final result.

Another potential source of systematic error is the renormalization functions which should be determined non-perturbatively. The scheme which is particularly convenient is the RI' defined as:

$$
Z_{q}=\left.\frac{1}{12} \operatorname{Tr}\left[\left(S^{L}(p)\right)^{-1} S^{\text {Born }}(p)\right]\right|_{p^{2}=\bar{\mu}^{2}},\left.\quad Z_{q}^{-1} Z_{\mathscr{O}} \frac{1}{12} \operatorname{Tr}\left[\Gamma_{\mathscr{O}}^{L}(p)\left(\Gamma_{\mathscr{O}}^{\text {Born }}(p)\right)^{-1}\right]\right|_{p^{2}=\bar{\mu}^{2}}=1 .
$$




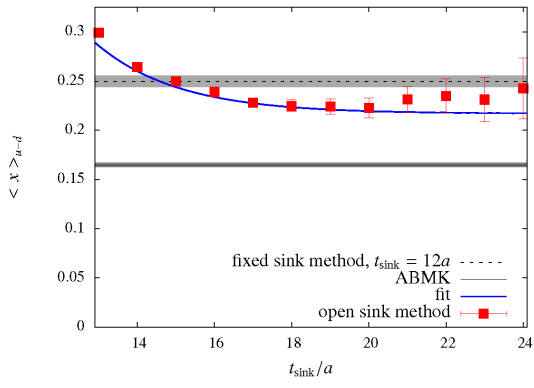

(a)

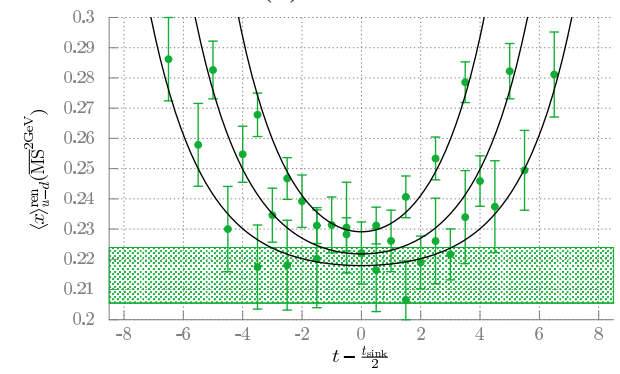

(c)

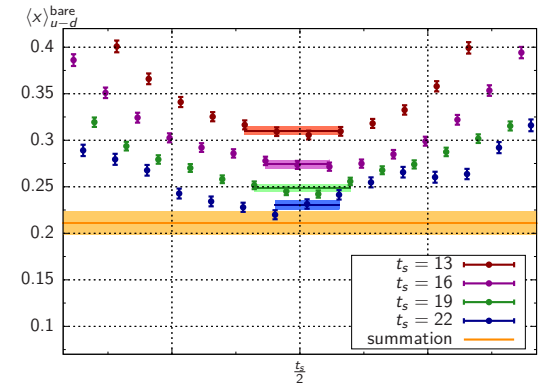

(b)

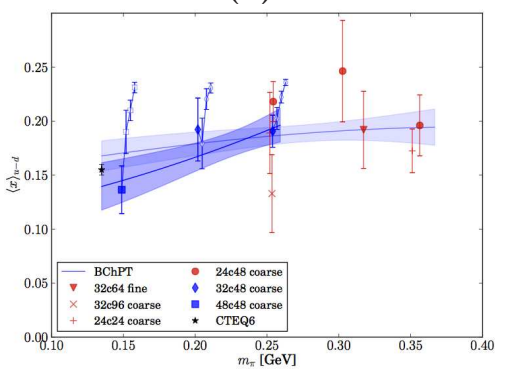

(d)

Figure 13: Studies for excited states contaminations using: (a) TMF at $m_{\pi}=373 \mathrm{MeV}$ (ETMC [29]), (b) Clover at $m_{\pi}=340 \mathrm{MeV}$ (MAINZ [51]), (c) Clover at $m_{\pi}=150 \mathrm{MeV}$ (RQCD [21]), and (d) Clover at $m_{\pi}=149 \mathrm{MeV}$ (LHPC [49]).

For the scheme and scale dependent operators such as $\langle x\rangle$, we need to convert to the $\overline{\mathrm{MS}}$ at $2 \mathrm{GeV}$, a step that also potentially can introduce systematic errors. We find that any error is insignificant when one utilizes the NNLO formulas. Lattice cut-off effects can also be ameliorated by using two alternative approaches: the first one employed by ETMC [52] is based on the subtraction of $\mathscr{O}\left(a^{2}\right)$ terms computed perturbatively [53], which improves the quality of the plateau (see left panel of Fig 14). The second approach employed by QCDSF [54] is the subtraction of the one-loop artifacts to all order in the lattice spacing, eliminating the slope of the plateau with respect to $(a p)^{2}$. It was shown that the complete subtraction works very well even at high scales where one might have expected the $\mathscr{O}\left(a^{2}\right)$ subtraction not to be sufficient.
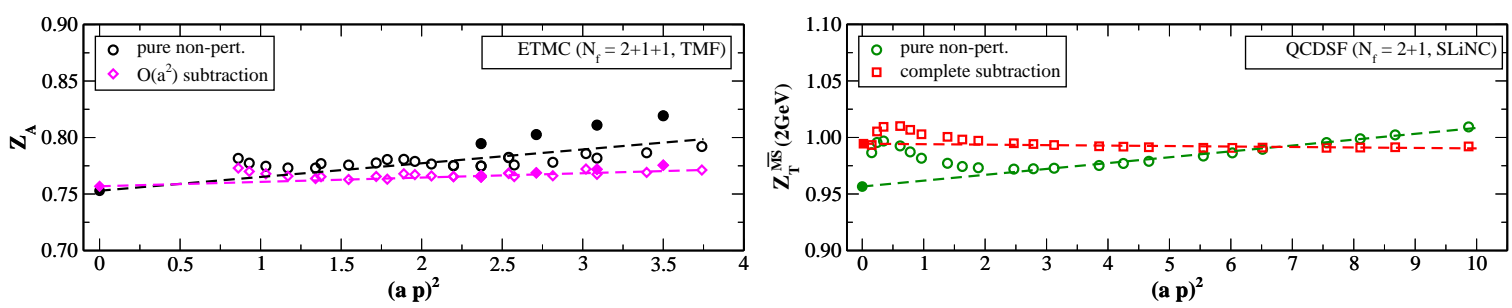

Figure 14: Left (Right): $Z_{A}[52]\left(Z_{T}[54]\right)$ as a function of the renormalization scale.

One may also control a priori the lattice artifacts by using momenta that have small nonLorentz invariant contributions: empirically $\left(\sum_{\rho} p_{\rho}^{4}\right)^{4} /\left(\sum_{\rho} p_{\rho}^{2}\right)^{2}\langle 0.4$. For example, the black filled circles shown in Fig. 14 for the case of $Z_{A}$ violate the above condition and are consistently higher than the rest. Note that, upon subtraction of the $\mathscr{O}\left(a^{2}\right)$ terms, the magenta points fall nicely on one line. Thus it is apparent that a combination of perturbative and non-perturbative methods can improve the determination of the renormalization factors. 


\subsection{Nucleon Spin}

A long-standing puzzle has been the spin fraction carried by the quarks in the nucleon, which is found to be about half of the total nucleon spin [55]. It was proposed that gluons in a polarized proton would carry a fraction of the spin, which however would be unnaturally large if it were to resolve the spin crisis. It is now understood that the resolution of this puzzle requires to take into account the non-perturbative structure of the proton. Using the lattice QCD formalism one can provide significant input towards understanding this open issue. The total nucleon spin is generated by the quark orbital angular momentum $\left(L^{q}\right)$, the quark spin $\left(\Sigma^{q}\right)$ and the gluon angular momentum $\left(J^{G}\right)$. The quark components are related to $g_{A}^{q}$ and the GFFs of the one-derivative vector at $Q^{2}=0$ :

$$
\frac{1}{2}=\sum_{q}\left(L^{q}+\frac{1}{2} \Delta \Sigma^{q}\right)+J^{G}, \quad J^{q}=\frac{1}{2}\left(A_{20}^{q}+B_{20}^{q}\right), \quad L^{q}=J^{q}-\Sigma^{q}, \quad \Sigma^{q}=g_{A}^{q} .
$$

Since we are interested in the individual quark contributions to the various components of the spin, one needs to consider the disconnected contributions.

The computation of disconnected diagrams using improved actions with dynamical fermions became feasible over the last years and for the proper renormalization of the individual quark and isoscalar contributions one should take into account the singlet operator. Non-perturbatively these are unknown (for a recent study using the Feynman-Hellmann approach, see Ref. [56]), but are expected to be small since in perturbation theory they first appear to two loops. Recent results by the Cyprus group [57] show that only the scalar and axial contributions survive to two loops. The results are general enough to be used by any combination between Wilson, Clover, SLiNC, TM fermions and any of the Symanzik improved gluons. The difference $Z_{\mathscr{O}}^{\text {singlet }}-Z_{\mathscr{O}}^{\text {non-singlet }}$ may be added to the non-perturbative non-singlet estimates for the Z-factors before using them to renormalize the disconnected contributions.
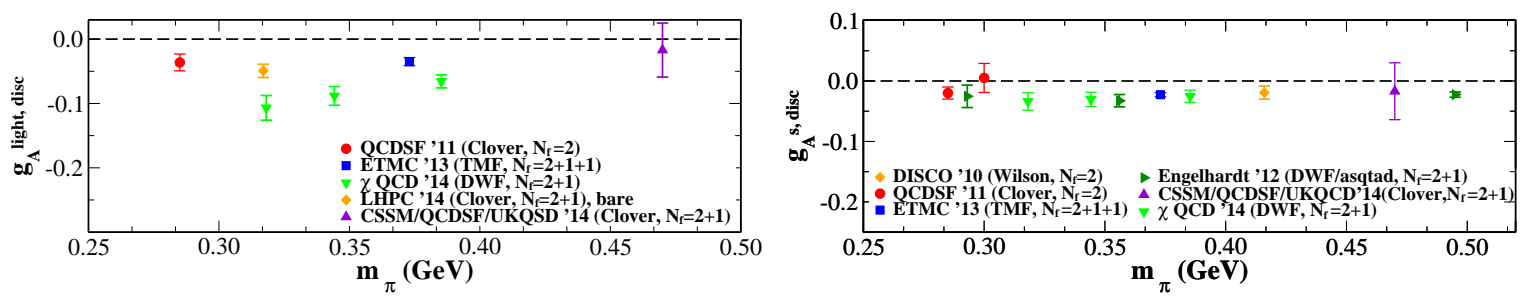

Figure 15: Disconnected contributions for $g_{A}^{q}$ for the light (left) and strange (right) quark contributions.

A number of results have appeared recently where the disconnected loop contributions to $g_{A}$ are evaluated as shown in Fig. 15. We observe a nice agreement among results using a number of methods both for the light $[8,10,58,37,59]$ as well as for the strange quark contributions $[7,8$, $10,9,37,59]$. For $g_{A}^{\text {light }}$ we find $\sim 10 \%$ contributions compared to the connected part that must be taken into account in the discussion of the spin carried by quarks in the proton. These contributions are negative and thus reduce the value of $g_{A}^{q}$. There is also a computation for the disconnected contribution to $\langle x\rangle$ using TM fermions [10] at $m_{\pi}=373 \mathrm{MeV}$, which is also of interest since it contributes to the spin. At this particular pion mass it was found to be compatible with zero.

In Fig. 16 we show results on the total spin, $J^{q}$. It is apparent that the u-quark exclusively carries the spin attributed to the quarks in the nucleon since $J^{d}$ is consistent with zero for all pion masses and lattice discretization schemes. The quark distribution to the intrinsic spin in also shown in Fig. 16. There is a nice agreement between the results at the physical pion mass using TM fermions [27] and the experimental values for both the u- and the d-quarks. In all the TMF data, 
the $Z_{A}^{\text {singlet }}$ is computed perturbatively [57] and has been included in the determination of the results. The disconnected contributions have been neglected from most data except for one TMF ensemble at $m_{\pi}=373 \mathrm{MeV}$. The effect is shown by the deviation of the filled blue square which has no disconnected contributions from the violet triangle, which include them. Although the effect is small it is larger than the statistical error and thus one needs to take them into acccount. The lattice results thus corroborate the missing spin contribution arising from the quarks. Whether gluonic degrees of freedom are responsible for the other half is debatable and remains an open question.
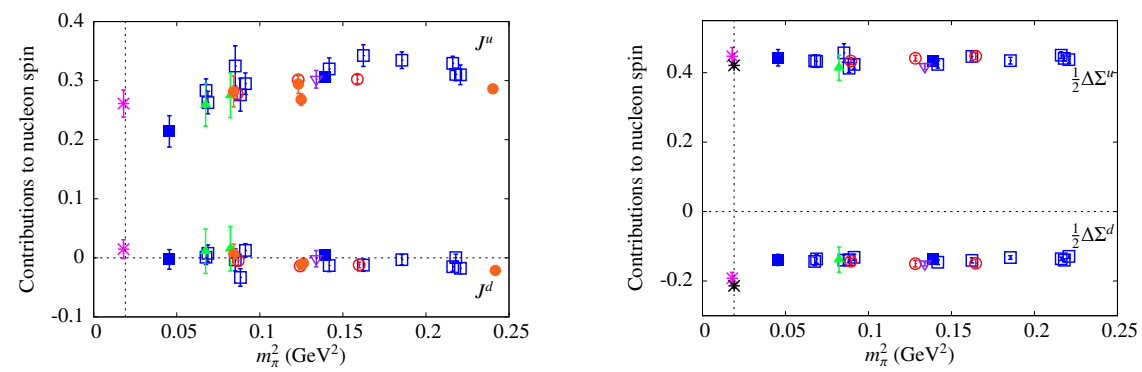

Figure 16: The total spin, $J^{q}$, and the quark spin, $\Sigma^{q}$, carried by the up and down quarks. The lattice data correspond to: $N_{f}=2+1$ DWF and DWF on asqtad (LHPC [46]), $N_{f}=2$ Clover (QCDSF/UKQCD [48]), $N_{f}=2 \mathrm{TMF}\left(\right.$ ETMC [45]) $N_{f}=2+1+1 \mathrm{TMF}$ (ETMC [24]) $N_{f}=2 \mathrm{TMF}$ with Clover (ETMC [27]).

\section{Hyperon Form Factor}

\subsection{Electromagnetic Form Factors}

The form factors of baryons other than the nucleon are poorly known and not amenable to measurements. Thus lattice QCD can provide significant input on these quantities. In a recent work of CSSM/QCDSF/UKQCD $[60,61]$ the hyperon EM FFs were computed at various values of $m_{\pi}$. By setting the electric charge of the sea quarks to zero, disconnected quark loops are completely removed. The authors perform independent chiral fits to the data at each value of $Q^{2}$, and thus, the coefficients in the chiral expansion are considered to be the chiral limit at fixed $Q^{2}$. This procedure is shown for the $G_{E}$ and $G_{M}$ of the $\Sigma$ in Fig. 17a, where the chirally extrapolated results are shown with black points. For the case of the proton, the authors find agreement with the experimental values after performing a chiral extrapolation using their approach.

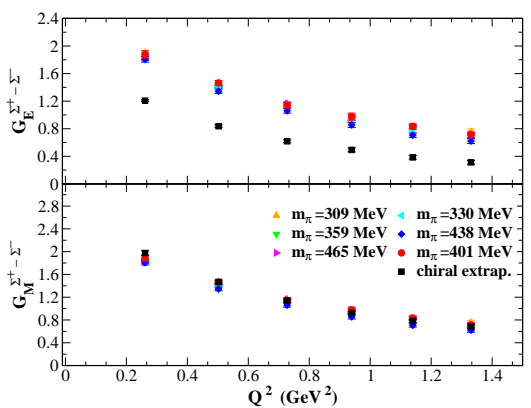

(a)

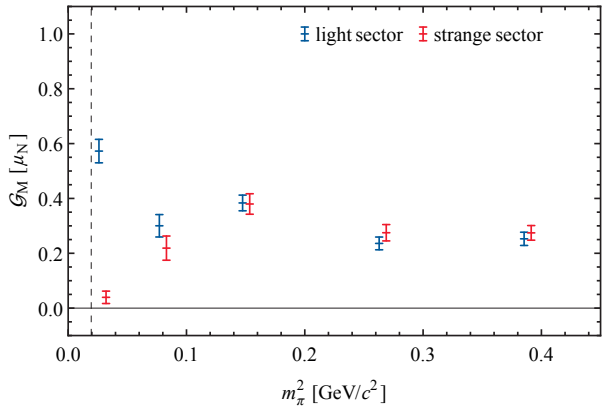

(b)

Figure 17: (a). $G_{E}^{\Sigma}$ and $G_{M}^{\Sigma}$ as a function of $Q^{2}$ for $m_{\pi}$ in the range of $309 \mathrm{MeV}$ to $465 \mathrm{MeV}$ along with the chiral extrapolation [60, 61]. (b). The light and strange quark contributions to $G_{M}$ of the $\Lambda(1405)[62,63]$. 
CSSM has studied the magnetic FF of the lowest lying excitation of the $\Lambda$ baryon, $\Lambda(1405)$, which despite its quark structure that includes a strange quark is lighter than other excited spin $1 / 2$ baryons. There are speculations that $\Lambda(1405)$ is a superposition of molecular meson-baryon states where the $\pi \Sigma$ and $\bar{K} N$ channels play a significant role. Study of the strange contribution to $G_{M}$ may shed light on the internal structure of this particle, since if it is an $\bar{K} N$ bound state, then the s-quark is confined in a spin zero kaon with no preference in the spin orientation, and thus, $G_{M} \rightarrow 0$. The lattice data for this study were extracted using a variational analysis for several values of the pion mass. Simulations very close to the physical point show a significant drop of $G_{M}^{s}$ which indeed becomes almost zero (see Fig. 17b). According to the authors, this is an indication that $\Lambda(1405)$ is dominated by a $\bar{K} N$ bound state.

\subsection{Axial Charges of Hyperons}

The axial charge of hyperons is another example where methods used in the nucleon sector can give predictions needed, for instance, as an input in chiral effective theories. On the lattice, the hyperon axial charges are extracted from the matrix element $\left.\left\langle B\left(p^{\prime}\right)\left|\bar{\psi}(x) \gamma_{\mu} \gamma_{5} \psi(x)\right| B(p)\right\rangle\right|_{q^{2}=0}$.

In Fig. 18 we show $g_{A}$ for $\Sigma$ and $\Xi$ for different discretizations as a function of $m_{\pi}$. Results using TMF probe the small pion mass region reaching the physical point. There is no strong pion mass dependence even for pion masses close to the phsyical value. This behaviour is also observed in the case of other baryons. In the same figure we show the SU(3) symmetry breaking $\delta_{S U(3)}=g_{A}^{N}-g_{A}^{\Sigma}+g_{A}^{\Xi}$ versus $x=\left(m_{K}^{2}-m_{\pi}^{2}\right) /\left(4 \pi^{2} f_{\pi}^{2}\right)$. As expected it increases quadratically with $x$. Simulations at the physical point with TMF [27] show about $15 \%$ SU(3) flavor breaking.
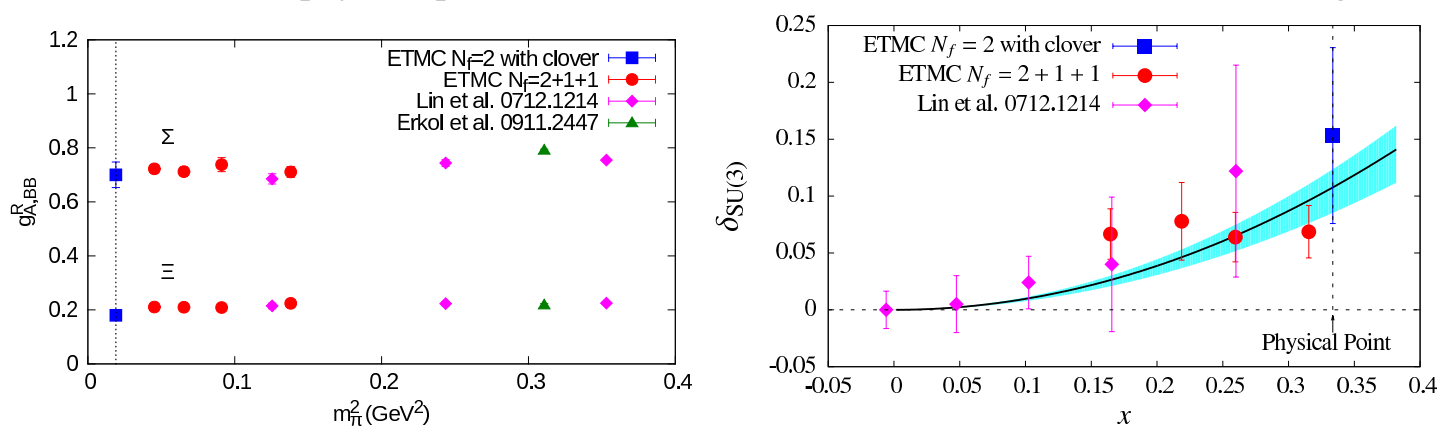

Figure 18: Left: $g_{A}$ for $\Sigma$ and $\Xi$ as a function of $m_{\pi}^{2}$ ([27] and references therein). Right: SU(3) breaking parameter $\delta_{S U(3)}$ versus $x$.

\section{Mesons}

Apart from studies of baryon structure, there are recent developments in the meson sector, which however we will only summarize here, focusing on the pion and the $\rho$-meson.

\subsection{Pion Quark Distribution Function}

The pion is a Goldstone boson playing a central role in chiral symmetry breaking with many consequences in nuclear and particle physics phenomena. Although it is consider a simple bound state its internal structure in not well studied. In fact, information about pion parton distribution functions (PDFs) rely on input from nucleon PDFs. Thus, Lattice QCD is in a unique position to provide results from first principles. 
In a recent study with $\mathrm{TMF}$ the momentum fraction $\langle x\rangle$, in the pion is computed using the operator $\mathscr{O}_{44}$

$$
\mathscr{O}_{44}(x)=\frac{1}{2} \bar{u}(x)\left[\gamma_{4} \stackrel{\leftrightarrow}{D}_{4}-\frac{1}{3} \sum_{k=1}^{3} \gamma_{k} \stackrel{\leftrightarrow}{D}_{k}\right] u(x), \quad\langle x\rangle_{\pi^{+}}^{\text {bare }}=\frac{1}{2 m_{\pi}^{2}}\left\langle\pi, \overrightarrow{0}\left|\mathscr{O}_{44}\right| \pi, \overrightarrow{0}\right\rangle
$$

The matrix element of this operator is non-zero at $Q^{2}=0$ and thus $\langle x\rangle$ can be easily extracted without requiring extrapolating the results to zero momentum transfer. A stochastic time source is utilized allowing high statistical accuracy at small computational cost. In the left panel of Fig. 19 the momentum fraction is plotted against $m_{\pi}^{2}$ for Wilson TMF and Clover fermions. There is quite a spread in the results obtained using the different discretizations that may point to large lattice artifacts that need to be investigated. Lattice results close to the physical point underestimate the phenomenological value of $\langle x\rangle_{\pi^{+}}=0.0217(11)$ [64]. A comprehensive study of lattice artifacts is called for in order to understand the observed discrepancies in the lattice data.
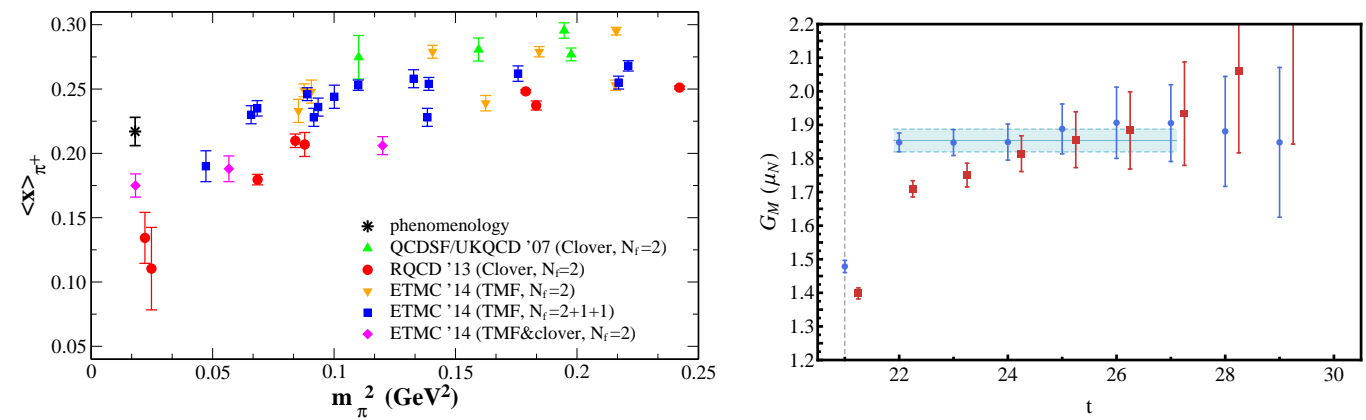

Figure 19: Left: $\langle x\rangle_{\pi^{+}}$as a function of $m_{\pi}^{2}$ for: $N_{f}=2$ Clover (QCDSF/UKQCD [65], RQCD [66]), $N_{f}=2$ TMF (ETMC [67]), $N_{f}=2+1+1$ TMF (ETMC [68]), $N_{f}=2$ TMF with Clover (ETMC [68]). Right: Plateau for $G_{M}$ of the $\rho$-meson [69] for the standard (red points) and variational (blue points) methods.

\section{2 $\rho$-meson Electromagnetic Form Factor}

The EM of the $\rho$-meson are computed by the CSSM collaboration using $N_{f}=2+1$ Clover fermions [69]. Since the $\rho$ is a resonance its form factors are not known. The $\rho$-meson matrix element of the EM current can be decomposed into a charge, magnetic and quadrupole form factor, denoted by $G_{C}, G_{M}$ and $G_{Q}$, respectively. A variational approach has been utilized and was found to separate excited states effectively. The technique is applied on a set of operators built from various source and sink smearings, applied to the $\rho$ interpolating field $\chi_{\rho}^{i}(x)=\bar{d}(x) \gamma^{i} u(x)$. Four levels of Gaussian smearing were employed, thus, a $4 \times 4$ correlation matrix was analyzed. The authors find substantial improvement in the determination of $G_{M}$ and $G_{Q}$ using the variational approach. In the right plot of Fig. 19 we show the results for $G_{M}$ using the standard method and the variational method. As can be seen, the plateau using the variational method begin only one time slice afte the current insertion, as compared to the standard method where no clear plateaus were observed.

\section{Conclusions and Perspectives}

Several major improvements in algorithm and techniques coupled with increase in the computational power have allowed lattice QCD simulations with light quark masses fixed to their physical value. Although this is a big achievement, many challenges lie ahead: development of appropriate 
algorithms to reduce the statistical errors at reduced cost, understanding how to treat unstable particles and resonances, inclusion of multi-particle states and computing accurately observables that probe beyond the standard model physics are some of them.

For hadron structure, simulations at different lattice spacings and larger volumes are crucial for a proper study of lattice artifacts in order to provide reliable results at the continuum limit. Such studies require an accuracy which is difficult to achieve with standard methods. All-ModeAveraging or other noise reduction techniques are thus essential in order to settle some of the long-standing discrepancies reviewed in this talk.

Similarly techniques developed for the computation of disconnected quark loop diagrams, such as the truncated solver method [70] need to be improved since they become inefficient at the physical point [71]. Hierarchical probing proved to be very promising in the evaluation of the electromagnetic form factors at pion mass of $\sim 300 \mathrm{MeV}$ is doubtful that it can work so well at the physical point. Thus, new ideas will be needed to compute disconnected contributions to hadron structure to an accuracy of a few percent. This is a real challenge if lattice QCD wants to contribute in the debate on the charge radius of the proton. Utilization of new computer architectures such as GPUs has proved essential for the evaluation of disconnected diagrams and this is a direction that should be pursued in the future.

Other open issues such as the nucleon spin may need the computation of challenging quantities such as the gluonic contributions, which beyond gauge noise, are difficult to renormalized mixing with other operators. Extending the formalism from the nucleon to other baryons will be another challenge since most of these particles decay strongly.

Despite the aforementioned challenges lattice QCD has entered a new era in terms of simulations. Having gauge configurations at the physical point has eliminated one of the systematic error that was inherent in all lattice calculations in the past, namely the difficult to quantify systematic error due to the chiral extrapolation in particular for the baryon-sector. This has opened the way to address a number of challenging systems directly at the physical point. This hold the promise to resolve discrepancies on benchmark quantities like $g_{A}$ and reliably compute quantities relevant for revealing possible new physics.

Acknowledgments: I would like to thank all those who have shared with me their recent work (listed in alphabetic order): C. Alexandrou, G. Bali, M. Gong, R. Gupta, C. Kallidonis, G. Koutsou, D. Leinweber, K.-F. Liu, S. Meinel, S. Ohta, B. Owen, H. Panagopoulos, Th. Rae, Ph. Shanahan, C. Urbach, Y.-B. Yang, and J. Zanotti. I greatly appreciate the fruitful discussions I had with my collaborator C. Alexandrou while preparing this talk. Last but not least I thank A. Abdel-Rehim, K. Hadjiyiannakou, G. Koutsou for discussions on technical oriented topics. The speaker is supported

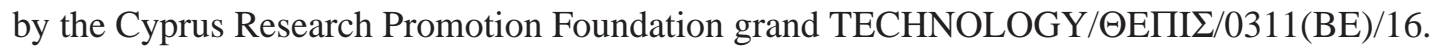

\section{References}

[1] http://www.jlab.org/

[2] A. Antognini, et al., Science 339 (2013) 417.

[3] P. J. Mohr, B. N. Taylor, D. B. Newell, Rev. Mod. Phys. 84, 1527 (2012) [arXiv:1203.5425].

[4] R. Pohl et al., Nature 466 (2010) 213. 
[5] R. S. Van Dyck, P. B. Schwinberg, H. G. Dehmelt, Phys. Rev. Lett. 59 (1987) 26 ; H. N. Brown et al. [Muon g-2], Phys. Rev. Lett. 86 (2001) 2227.

[6] D. d'Enterria, G. G. da Silveira, Phys. Rev. Lett. 111, 080405 (2013) [arXiv:1305.7142].

[7] R. Babich, R. C. Brower, M. A. Clark, G. T. Fleming, J. C. Osborn, C. Rebbi, D. Schaich, Phys. Rev. D 85, 054510 (2012) [arXiv:1012.0562].

[8] G. S. Bali et al. [QCDSF Collaboration], Phys. Rev. Lett. 108, 222001 (2012) [arXiv:1112.3354].

[9] M. Engelhardt, Phys. Rev. D 86, 114510 (2012) [arXiv:1210.0025].

[10] A. Abdel-Rehim, C. Alexandrou, M. Constantinou, V. Drach, K. Hadjiyiannakou, K. Jansen, G. Koutsou, A. Vaquero, Phys. Rev. D 89, 034501 (2014) [arXiv:1310.6339].

[11] T. Yamazaki et al. [RBC/UKQCD Collaboration], Phys. Rev. Lett. 100, 171602 (2008) [arXiv:0801.4016].

[12] T. Yamazaki, Y. Aoki, T. Blum, H. W. Lin, S. Ohta, S. Sasaki, R. Tweedie, J. Zanotti, Phys. Rev. D 79, 114505 (2009) [arXiv:0904.2039].

[13] S. Ohta [RBC/UKQCD Collaboration], PoS LATTICE 2013274 (2013) [arXiv:1309.7942].

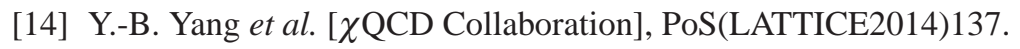

[15] J. D. Bratt et al. [LHP Collaboration], Phys. Rev. D 82, 094502 (2010) [arXiv:1001.3620].

[16] C. Alexandrou et al. [ETM Collaboration], Phys. Rev. D 83, 045010 (2011) [arXiv:1012.0857].

[17] D. Pleiter et al. [QCDSF/UKQCD Collaboration], PoS LATTICE 2010, 153 (2010) [arXiv:1101.2326].

[18] S. Capitani, M. Della Morte, G. von Hippel, B. Jager, A. Juttner, B. Knippschild, H. B. Meyer, H. Wittig, Phys. Rev. D 86, 074502 (2012) [arXiv:1205.0180].

[19] R. Horsley, Y. Nakamura, A. Nobile, P. E. L. Rakow, G. Schierholz , J. M. Zanotti, Phys. Lett. B 732, 41 (2014) [arXiv:1302.2233].

[20] G. S. Bali et al., PoS LATTICE 2013290 (2013) [arXiv:1311.7041].

[21] G. S. Bali et al. [RQCD Collaboration], PoS(LATTICE2014)136.

[22] J. Green, M. Engelhardt, S. Krieg, J. Negele, A. Pochinsky, S. Syritsyn, PoS LATTICE 2012, 170 (2012) [arXiv:1211.0253].

[23] B. J. Owen, J. Dragos, W. Kamleh, D. B. Leinweber, M. S. Mahbub, B. J. Menadue, J. M. Zanotti, Phys. Lett. B 723, 217 (2013) [arXiv:1212.4668].

[24] C. Alexandrou, M. Constantinou, S. Dinter, V. Drach, K. Jansen, C. Kallidonis, G. Koutsou, Phys. Rev. D 88, 014509 (2013) [arXiv:1303.5979].

[25] T. Bhattacharya, S. D. Cohen, R. Gupta, A. Joseph, H. W. Lin, B. Yoon, Phys. Rev. D 89, 094502 (2014) [arXiv:1306.5435].

[26] R. Gupta et al. [PNDME Collaboration], PoS(LATTICE2014)152.

[27] C. Alexandrou et al. [ETM Collaboration], PoS(LATTICE2014)151.

[28] J. Beringer et al., Particle Data Group, Phys. Rev. D 86, 010001 (2012).

[29] S. Dinter, C. Alexandrou, M. Constantinou, V. Drach, K. Jansen, D. B. Renner, Phys. Lett. B 704, 89 (2011) [arXiv:1108.1076].

[30] A. J. Chambers et al., Phys. Rev. D 90, 014510 (2014) [arXiv:1405.3019].

[31] E. Shintani, R. Arthur, T. Blum, T. Izubuchi, C. Jung, C. Lehner, arXiv:1402.0244.

[32] S. Ohta [RBC/UKQCD Collaboration], PoS(LATTICE2014)149.

[33] J. R. Green, J. W. Negele, A. V. Pochinsky, S. N. Syritsyn, M. Engelhardt, S. Krieg, Phys. Rev. D 90, 074507 (2014) [arXiv:1404.4029].

[34] A. Abdel-Rehim et al. [ETM Collaboration], PoS(LATTICE2014)148.

[35] J. J. Kelly, Phys. Rev. C70, 068202 (2004). 
[36] M. Lin, PoS LATTICE 2013, 275 (2014) [arXiv:1401.1476].

[37] S. Meinel et al. [LHP Collaboration], PoS(LATTICE2014)139.

[38] A. Stathopoulos, J. Laeuchli, K. Orginos, SIAM J. Scientific Computing (2013). arXiv:1302.4018.

[39] M. Lin [RBC/UKQCD Collaboration], PoS LATTICE 2012, 172 (2012) [arXiv:1303.0022].

[40] C. Alexandrou, M. Brinet, J. Carbonell, M. Constantinou, P. A. Harraud, P. Guichon, K. Jansen, T. Korzec, M. Papinutto, Phys. Rev. D 83, 094502 (2011) [arXiv:1102.2208].

[41] S. N. Syritsyn et al. [LHP Collaboration], Phys. Rev. D 81, 034507 (2010) [arXiv:0907.4194].

[42] S. Collins et al., Phys. Rev. D 84, 074507 (2011) [arXiv:1106.3580].

[43] S. Capitani, M. Della Morte, G. von Hippel, B. Jager, B. Knippschild, H. B. Meyer, T. D. Rae, H. Wittig, PoS LATTICE 2012, 177 (2012) [arXiv:1211.1282].

[44] C. Alexandrou et al. [ETM Collaboration], PoS(LATTICE2014)144.

[45] C. Alexandrou, J. Carbonell, M. Constantinou, P. A. Harraud, P. Guichon, K. Jansen, C. Kallidonis, T. Korzec, M. Papinutto, Phys. Rev. D 83, 114513 (2011) [arXiv:1104.1600].

[46] S. N. Syritsyn, J. R. Green, J. W. Negele, A. V. Pochinsky, M. Engelhardt, P. Hagler, B. Musch, W. Schroers, PoS LATTICE 2011, 178 (2011) [arXiv:1111.0718].

[47] Y. Aoki, T. Blum, H. W. Lin, S. Ohta, S. Sasaki, R. Tweedie, J. Zanotti, T. Yamazaki, Phys. Rev. D 82, 014501 (2010) [arXiv:1003.3387].

[48] A. Sternbeck et al., PoS LATTICE 2011, 177 (2011) [arXiv:1203.6579].

[49] J. R. Green, M. Engelhardt, S. Krieg, J. W. Negele, A. V. Pochinsky, S. N. Syritsyn, Phys. Lett. B 734, 290 (2014) [arXiv:1209.1687].

[50] S. Alekhin, J. Blumlein, S. Klein, S. Moch, Phys. Rev. D 81, 014032 (2010) [arXiv:0908.2766].

[51] B. Jäger, T. D. Rae, S. Capitani, M. Della Morte, D. Djukanovic, G. von Hippel, B. Knippschild, H. B. Meyer, H. Wittig, PoS LATTICE 2013 272, (2013) [arXiv:1311.5804].

[52] C. Alexandrou, M. Constantinou, T. Korzec, H. Panagopoulos, F. Stylianou, Phys. Rev. D 86, 014505 (2012) [arXiv:1201.5025].

[53] M. Constantinou, V. Lubicz, H. Panagopoulos, F. Stylianou, JHEP 0910, 064 (2009) [arXiv:0907.0381].

[54] M. Constantinou, R. Horsley, H. Panagopoulos, H. Perlt, P. E. L. Rakow, G. Schierholz, A. Schiller, J. M. Zanotti, arXiv:1408.6047.

[55] J. Ashman et al. (European Muon Collaboration), Phys. Lett. B206, 364 (1988).

[56] A. J. Chambers, R. Horsley, Y. Nakamura, H. Perlt, P. E. L. Rakow, G. Schierholz, A. Schiller, J. M. Zanotti, arXiv:1410.3078.

[57] M. Constantinou, M. Hadjiantonis, H. Panagopoulos, PoS(LATTICE2014)298.

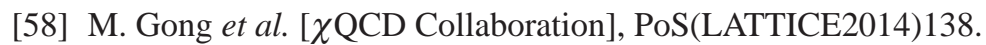

[59] J. Zanotti et al. [CSSM/QCDSF/UKQCD Collaboration], PoS(LATTICE2014)165.

[60] P. E. Shanahan et al., Phys. Rev. D 89, 074511 (2014) [arXiv:1401.5862].

[61] P. E. Shanahan et al., Phys. Rev. D 90, 034502 (2014) [arXiv:1403.1965].

[62] B. J. Menadue, W. Kamleh, D. B. Leinweber, M. S. Mahbub, Phys. Rev. Lett. 108, 112001 (2012) [arXiv:1109.6716].

[63] D. Leinweber et al. [CSSM Collaboration], PoS(LATTICE2014)094.

[64] K. Wijesooriya, P. E. Reimer and R. J. Holt, Phys. Rev. C 72, 065203 (2005) [nucl-ex/0509012].

[65] D. Brommel et al. [QCDSF/UKQCD Collaboration], PoS(LATTICE)2007, 140 (2007).

[66] G. S. Bali, S. Collins, B. Gläßle, M. Göckeler, N. Javadi-Motaghi, J. Najjar, W. Söldner, A. Sternbeck, PoS LATTICE 2013, 447 (2013) [arXiv:1311.7639].

[67] R. Baron et al. [ETM Collaboration], PoS LAT 2007, 153 (2007) [arXiv:0710.1580].

[68] A. Abdel-Rehim et al. [ETM Collaboration], PoS(LATTICE2014)119.

[69] B. Owen et al. [CSSM Collaboration], PoS(LATTICE2014)159.

[70] G. S. Bali, S. Collins, A. Schäfer, Comput. Phys. Commun. 181, 1570 (2010) [arXiv:0910.3970].

[71] C. Alexandrou et al. [ETM Collaboration], PoS(LATTICE2014)140. 